\title{
Neurocysticercosis Presenting as Complex Partial Seizures
}

\author{
Deep Hathi ${ }^{1}$, Ayush Somani ${ }^{2}$, Anusha Gupta ${ }^{3}$, Sourya Acharya ${ }^{4}$ \\ ${ }^{1}$ Department of Medicine, Datta Meghe Institute of Medical Sciences (Deemed to be University), Sawangi, Meghe, \\ Wardha, Maharashtra, India. ${ }^{2}$ Department of Medicine, Datta Meghe Institute of Medical Sciences (Deemed to be \\ University), Sawangi, Meghe, Wardha, Maharashtra, India. ${ }^{3}$ Department of Medicine, Datta Meghe Institute of Medical \\ Sciences (Deemed to be University), Sawangi, Meghe, Wardha, Maharashtra, India. ${ }^{4}$ Department of Medicine, Datta \\ Meghe Institute of Medical Sciences (Deemed to be University), Sawangi, Meghe, Wardha, Maharashtra, India.
}

\section{INTRODUCTION}

Neurocysticercosis (NCC) occurs in 60-90 percent of cases when parasite disease cysticercosis affects the central nervous system. This disease occurs when an organism becomes the intermediate host to the larvae form of Taenia solium, the pork tapeworm. The clinical features, diagnostic modalities, and management of the condition all differ depending on the position and quantity of cysts, whether the parasite is functional and living or dormant and died, as well as the immune response of each person to the disease. The most common clinical feature when central nervous system is affected is seizures, and the most common seizure types being generalized tonic-clonic seizures seen in $30-60 \%$ patients followed by simple partial seizures seen in $8-50 \%$ patients. Complex partial seizures (CPS) are the rare type of seizure presentation seen in $2-20 \%$ patients. We present a case of 30 year old male who presented with complex partial seizures and severe bilateral headaches. Magnetic resonance imaging was done which showed multiple ring enhancing lesion throughout the brain parenchyma suggestive of neurocysticercosis. Ultrasound of subcutaneous nodules also showed presence of scolex which confirmed our diagnosis. He was started on albendazole, steroids in tapering doses, clobazam and levetiracetam. He was symptom free at 2 months after follow-up.

NCC is a disorder of CNS caused due to infection with the larval stage of the intestinal pork tapeworm T. solium. Through the faecal-oral route, individuals become infected with the larval form of the parasite by ingesting parasite eggs from a person with an adult tapeworm in the intestine.(1) The larval form of the organism can be present in almost every human tissue,(2) however, it has an affiliation to the brain tissue where it forms a cyst enclosing the parasite. $(3,4)$

The encysted parasite progresses through three distinct phases of evolution

1. The active phase: in this phase, the parasites are alive.

2. The transitional phase: in this phase, the parasite start to degenerate and immune system starts of the host starts acting.

3. The inactive phase: the parasite are died but a calcified nodule is seen.(5)

NCC may be the most heterogeneous CNS disease with a wide range of clinical manifestations.(6) However, seizure is the most common symptom. Seizures are the most common manifestation of cysts formation in brain parenchyma, ${ }^{(3)}$ and can be seen in all 3 phases mentioned above.(4)
Corresponding Author: Dr. Sourya Acharya,

Professor,

Department of Medicine,

Datta Meghe Institute of Medical Sciences (Deemed to be University), Sawangi Meghe, Wardha, Maharashtra, India.

E-mail: souryaacharya74@gmail.com

DOI: $10.14260 / j e m d s / 2020 / 241$

Financial or Other Competing Interests: None.

How to Cite This Article:

Hathi D, Somani A, Gupta A, et al. Neurocysticercosis presenting as complex partial seizures. J. Evolution Med. Dent. Sci. 2020;9(13):1116-1118, 10.14260/jemds/2020/241

Submission 04-12-2019, Peer Review 06-03-2020, Acceptance 12-03-2020, Published 30-03-2020.

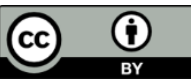


In the transitional stage, seizures associated with cysts, are characterized by oedema surrounding the degenerating cyst, are known to be symptomatic or acute. Active or inactive cyst-related seizures are considered unprovoked.(4) It has been hypothesized that the chronic epileptic foci which is resulting from parietal gliosis(7) or scarring ${ }^{(8)}$ is responsible for inactive cysts causing seizures. The most common forms of seizures recorded in patients with NCC are generalized seizures and partial seizures with secondary generalization; while only a few report with complex partial seizures. $(4,8)$ Del Brutto et al in 1996 studied the pattern of seizure in 203 patients with NCC, and concluded that in both categories (single and multiple NCC lesions) the commonest form of seizure presentation was GTCS (52.60\%) followed by simple partial seizures, and least common variety being complex partial seizures (4.3\%).(9) The explanation for such low incidence of complex partial seizures in NCC patients could be due to large proportion of NCC patients with cysts in the parietal and frontal lobes and the comparatively few patients with cyst in temporal lobe.(7) We report a case of a middle-aged men who presented with a prolonged complex partial seizure and bi-frontal headaches.

\section{PRESENTATION OF CASE}

A 30-year-old male presented to casualty with complains of 1 episode of seizure in morning. He also complains of bilateral severe headache since 5 days. History of similar seizure episode was present 3 days ago. Patient had headache to begin with following which he had confusion, disorientation and seizure at the end of each episode. There were presence of atypical automatism in form of lips smacking and grunting sounds as described by the relatives. Following this confused state the patient was found lying on the floor unresponsive, staring, with rhythmic clonic movements of the left face, arm, and leg. Each episode lasted for 45 seconds to 1 minute and he had such 3-4 episodes before he was brought to hospital. He was brought to the emergency department of our hospital where intravenous diazepam was given. The seizure subsided within minutes, following which the patient was admitted in ICU for further investigation for cause of seizures.

He denied any past history of head trauma/Diabetes mellitus/Tuberculosis/Hypertension/any congenital disease /febrile seizures or family history of seizure disorder. The patient mainly eats non-vegetarian food and gave history of eating uncooked pork once in the past. He also gave history of taking fresh vegetables (cooked, uncooked) and fruits.

His local examination revealed multiple numerous nodules of varying sizes over abdomen and calf muscles. Systemic examination revealed no abnormality. His later testing of higher cognitive functions was found to be intact.

The magnetic resonance imaging (MRI) showed multiple well defined ring enhancing lesions throughout the brain parenchyma suggesting possibility of neurocysticercosis. Ultrasonography was performed at local site of subcutaneous nodules showed multiple well encapsulated hypoactive lesion with central echogenic nodule s/o scolex, which were in favour of cysticercosis in subcutaneous plane.

The patient was started on inj. Dexamethasone, albendazole, clobazam and levetiracetam and was discharged with levetiracetam $500 \mathrm{mg}$ BD, Clobazam $10 \mathrm{mg}$ at bedtime, Albendazole $400 \mathrm{mg}$ BD for 2 weeks (total 3 weeks), along with
Prednisolone in tapering doses. Patient was also given naproxen BD for headache. Patient was followed up after 2 weeks in OPD. He was advised to continue levetiracetam and Clobazam. He did not have any seizure episode till his 2nd follow-up after 2 months.
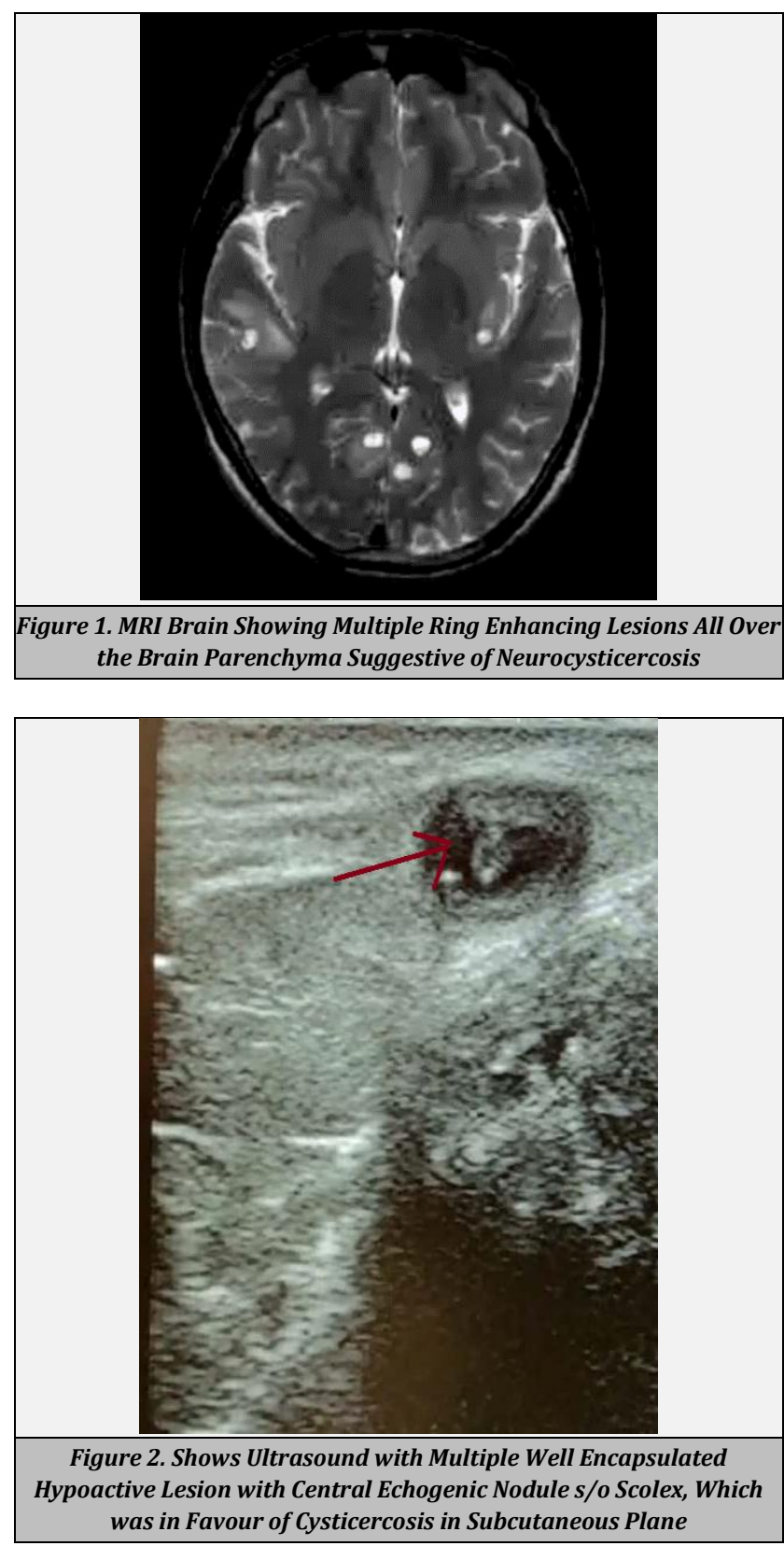

\section{DISCUSSION}

There is no definite age group for presentation as NCC. Samir Vora et al. carried out a cross sectional study in Goan village on 450 patients to study the sero prevalence of cysticercosis using ELISA test. The study concluded with prevalence of $22.4 \%$, which is considered as one of the highest in the world. (10)

Neurocysticercosis presenting as complex partial seizure (CPS) is rare. CPS arises from the inferior frontal lobe or medial temporal lobe.(11) patients may also present with headache, intracranial hypertension, strokes, diplopia, neuropsychiatric disturbances, hydrocephalus.(9) Del Brutto et 
al. provided criteria for diagnosis of neurocysticercosis based on objective clinical, immunologic, imaging and epidemiologic data. "These include four categories of criteria stratified on the basis of their diagnostic strength, including the following: 1) absolute, 2) major, 3) minor and 4) epidemiologic". The criteria provides with two degrees of diagnosis: "1) definitive diagnosis, in patients who have one absolute criterion or in those who have two major plus one minor and one epidemiologic criterion; and 2) probable diagnosis, in patients who have one major plus two minor criteria, in those who have one major plus one minor and one epidemiologic criterion, and in those who have three minor plus one epidemiologic criterion".(12) patient in our case had one absolute criteria (histopathological), one major criteria (highly suggestive cystic lesion in CT scan) and one epidemiological criteria (coming from an endemic area). Hence, diagnosis of neurocysticercosis was definitive.

Therapeutic trials has shown albendazole to be superior than praziquantel in treatment of NCC.(13) The advantages of using Albendazole over praziquantel is that it has better CSF penetration, it is not costly and easily available and can be safely given with steroids.(14) There is possibility of exacerbation of symptoms when treated with albendazole and praziquantel simultaneously due to local inflammation after death of larvae. Hence, we need to administer steroids with these drugs to avoid these complications and it also helps to reduce oedema and Intracranial hypertension.

Various studies have shown that treatment with albendazole results in better clearing of cysticerci, reduces risk for recurrent episodes of seizures in these patients.(15) In our case, patient was symptom free after 21 days therapy. Even though in patients with NCC, seizure is the most common presenting symptom, however CPS are extremely rare which makes our case unique. Parasitic infestation causes NCC which has variable neurological presentation. The clinical features and treatment modalities of this parasitic infestation vary from person to person due to various factors discussed above, thus it is recommended that NCC should be kept as a possibility in any adult presenting as seizure in absence of obvious cause or in regions where it is endemic.

\section{REFERENCES}

[1] Singhi P, Ray M. Focal seizures with single small ringenhancing lesion. Semin Pediatr Neurol 1999;6(3):196201.
[2] Schantz PM, Moore AC, Munoz JL, et al. Neurocysticercosis in an Orthodox Jewish community in New York City. N Engl J Med 1992;327(10):692-5.

[3] Sciutto E, Fragoso G, Fleury A, et al. Taenia solium disease in humans and pigs: an ancient parasitosis disease rooted in developing countries and emerging as a major health problem of global dimensions. Microbes Infect 2000;2(15):1875-90.

[4] Carpio A, Escobar A, Hauser WA. Cysticercosis and epilepsy: a critical review. Epilepsia 1998;39(10):102540.

[5] Carpio A, Placencia M, Santillan F, et al. A proposal for classification of neurocysticercosis. Can J Neurol Sci 1994;21(1):43-7.

[6] Parker F, Hladky JP, Breton JO, et al. Cysticercoseracemeuse de la queue de cheval et arachnoiditekystique a propos de deuxcas. Neurochirurgie 1988;34(4):280-5.

[7] Chayasirisobhon S, Menoni R, Chayasirisobhon W, et al. Correlation of electroencephalography and the active and inactive forms of neurocysticercosis. Clin Electroencephalogr 1999;30(1):9-11.

[8] White AC. Neurocysticercosis: a major cause of neurological disease worldwide. Clin Infect Dis 1997;24(2):101-13.

[9] Del Brutto OH. Neurocysticercosis. Semin Neurol 2005;25(3):243-51.

[10] Vora SH, Motghare DD, Kulkarni MS, et al. Prevalence of human cysticercosis and taeniasis in Rural Goa, India. J Commun Dis 2008;40(2):147-50.

[11] Fauci AS, Kasper DL, Hauser SL, et al. Harrison's principles of internal medicine. 17th edn. McGraw Hill Education 2008.

[12] Del Brutto OH, Rajshekhar V, White AC, et al. Proposed diagnostic criteria for neurocysticercosis. Neurology 2001;57(2):177-83.

[13] Takayanagui OM, Jardim E. Therapy for neurocysticercosis. Comparison between albendazole and praziquantel. Arch Neurol 1992;49(3):290-4.

[14] García HH, Evans CAW, Nash TA, et al. Current consensus guidelines for treatment of neurocysticercosis. Clin Microbiol Rev 2002;15(4):747-56.

[15] Del Brutto OH, Roos KL, Coffey CS, et al. Meta-analysis: cysticidal drugs for neurocysticercosis: albendazole and praziquantel. Ann Intern Med 2006;145(1):43-51. 